\title{
CHARACTERIZATION OF THE LAND-COVER AND LAND-USE BY SHAPE DESCRITORS IN TWO AREAS IN PONTA GROSSA, PR, BR
}

\author{
S. R. Ribeiro ${ }^{1 *}$, T. M. Hamulak \\ ${ }^{1}$ Department of Geography, State University of Ponta Grossa, Brazil \\ *corresponding email author: selmar.aranha@gmail.com
}

KEY WORDS: GEOBIA, remote sensing data, Geographic Information Systems - GIS.

\begin{abstract}
:
This study aims to identify the spatial characteristics of the land use and occupation regarding shape (geometry) that these uses are presented in two (2) Landscape units of Hydrographic Basin Pitangui by satellite Geographic Object-Based Image Analysis (GEOBIA). The two (2) croppings of landscape units come from the IRS satellite - P6 (Indian Remote-Sensing Satellite) ResourceSat LISS III sensor. The preprocessings were performed, geometry and atmosphere correction, and the image became segmented in order to extract from it the spatial characteristics and identify the relationship between descriptors of the segments, which were generated from GEOBIA and the use, and occupation of the land. An relevant aspect of this study is that one (1) of landscape units is located in the First Paraná Plateau and the other in the Second Paraná Plateau where there are significant differences concerning geology. The GEOBIA allows segmenting the image by means of regions growth technique and it extracts a relational database considering the spectral, spatial (shape) and texture attributes of the image digital levels. Through this database, we used the descriptors of the spatial attribute (shape) to analyze through graphics and specialization of them with color, to check the relationship between these shape descriptors. It is possible to identify the landscape morphology with this analysis, where small areas do not have defined shapes and the larger areas with geometrically defined shapes. It highlights the importance of analyzing the landscape and its relations with the use and pattern recognition and also the highlight of GEOBIA on this issue which enables to corroborate by the descriptors the visual analysis that the interpreter performs allowing more security and less subjectivity.
\end{abstract}

\section{INTRODUÇÃO}

This study aims to identify the morphology of two landscape units by comparing the shape attribute descriptors, arising Geographic Object - Based Image Analysis (GEOBIA) and this comparison identify which are the archetypes of this attribute with the occupation of landscapes in question, taking into consideration their shape descriptors, to be identified so that ground settlement patterns that characterize both areas.

To ensure the accuracy of the data from this study, we used some preprocessing and after these processing; They were performed targeting and GEOBIA, thereby generating segments and relational database sufficient for the conduct of the study.

\subsection{Segmentation and GEOBIA}

In the Geographic Object or Based Image Analysis (GEOBIA), the important processing is the segmentation of the image. It refers to the process of object image formation that according to Woods and Gonzalez () is the first step in Digital Image Processing (PDI). It consists of dividing the image into homogeneous regions by similarity or discontinuity of algorithms

During the segmentation process, segments are generated or objects through the basic properties of intensity values (digital level) Similar according to the rule used. Vectors are generated taking into consideration the level of abstraction of the segments and also to the relational database on the quantity of processed segments containing the descriptors, spatial, spectral and texture, coming from each region. RIBEIRO, 2003; PRICHOA, 2012; ANTUNES 2014).

\subsection{Object-Oriented Analysis}

The same came up with the preprocessing segmentation, the primary objective of facilitating the domain of a particular area of study to reduce subjectivity mainly in digital classification. She developed GEOBIA, and the most popular algorithms are the Fractal Net Evolution Approach (FNEA) and Full Lambda Schedule Algorithm
(FLSA), designed by Crisp et. al. (2002), this last one used in this study.

Studies by Shapiro and Stockman (2001) indicate two main purposes for using the GEOBIA technique in the analysis: transform the image into fragments for later review and make the change to the representation using their spatial attributes, spectral and texture.

The spatial attributes of shape, have geometric characteristics of the segments, which are translated into the area, length, compactness, convexity strength, roundness, shape factor, stretchiness, rectangularity, main direction, major axis, minor axis, the number of holes, relationship area/ holes.

In this work, we used the attributed form, because of the prior visual analysis difference (photo interpretation) on the morphology of the local landscape.

\section{MATERIALS AND METHODS}

To carry out this work the software utilised were: ENVI 4.7 ENVI EX and ArcGIS 10.2, and the first to the GEOBIA and the last for the analysis of spatial attributes of the relational database. We used the IRS image - P6 Resource Sat Liss III sensor, four spectral bands namely: green, red, near infrared and mid-infrared, with a spatial resolution of $23,5 \mathrm{~m}$, for targeting, available for free on the site INPE (Institute National Space Research). The image corresponds to the scene 328 orbit 96 of the city of Ponta Grossa, of the 07 March 2012. And also the Orth image with a spatial resolution of $5 \mathrm{~m}$, the systematic mapping of the state of Paraná, scale 1: 50,000, SG22 XD - III made available by Parana City, to base and Resource Sat Liss III geometry correction.

Figure 1 shows the block diagram of the methodology used in this study. 


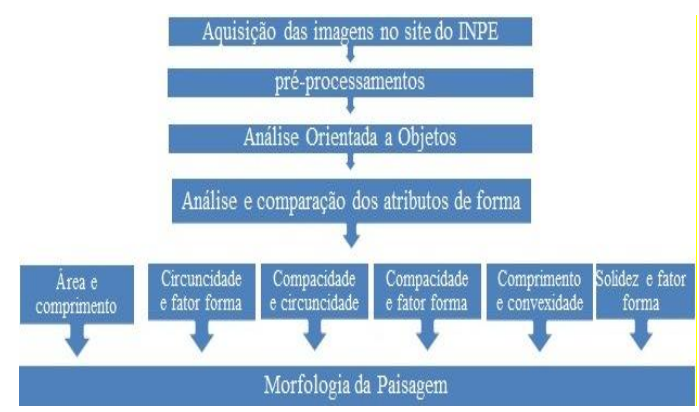

Figure 1. Shows the block diagram with the methodology used in this study.

The preprocessing of the geometric correction, inverse mapping and radiometric correction Dark Object OF Subtraction by key. These refer to the preliminary treatment of the raw data, to calibrate the image radiometry, mitigate the effects of the atmosphere, remove noise and correct geometric distortions arising in the image acquisition process. By GEOBIA is segmented-all spectral bands of images in true color composite false-color R (4) mid-infrared G (3) mid-infrared B (2) red, using the scale factor of 40 and the merger of 50 .

For the recognition of landscape forms, colored compositions were analyzed satellite Resource Satellite, Liss III sensor, and artificial images generated in the GIS of shape descriptors. For visual analysis used the true, false color, as mentioned, since the infrared band, especially next, enhance vegetation.

The artificial images of space descriptors formed by the database of shape descriptors in the GIS wereused in the legend's color scale that distributed the quantitative values and colors for the polygons. The colours are grouped into intervals arranged in the form of classes. The darker color tones highlight the descriptor relationships and the lighter tones generated between the space descriptors the scatter plots. In the representation of artificial images and scatter plots the data were normalized, i.e. the ratio was calculated by the descriptors. Normalization is relevant because of the numerical, difference of descriptors is significant.

\section{RESULTS}

Figure 2 represents the image clippings ResourceSat LISS III, truefalse color- colorful combination R (red) G (near infrared) B (midIR); the first and second unit landscape. Being the first area located at coordinates $603104.697239027 .62 \mathrm{E}$ and $\mathrm{N}$ and the second located at coordinates $583634.727234839 .68 \mathrm{E}$ and N. Located in South America, Brazil, in the state of Paraná in the city of Ponta Grossa.
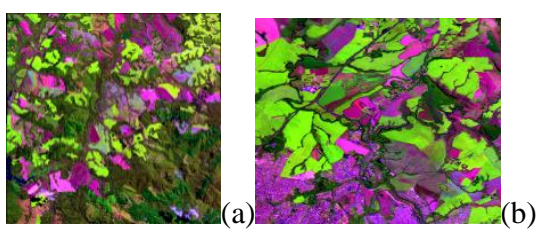

Figure 2. Images that represent the landscape units used in the study first landscape unit (a) located between the coordinates 603,104.69 E 7,239,027.62 $\mathrm{N}$ and second landscape (b) located in coordinates $583634,72 \mathrm{E}$ and $7234839.68 \mathrm{~N}$.

The pre-processing performed in this study were: geometric transformation and the radiometric correction. The geometric correction was obtained employing square error of $6.2 \mathrm{~m}$. The results of GEOBIA with the descriptors are shown in Figures 3 and 4.

Figure 3 (a) and (b) show the segmentation of the first landscape unit (a) and the second landscape unit (b). In Figure 3 (a) it is emphasized that the major joined smaller segments, adding areas to the scale were super-targeted. Note that these areas correspond to areas far as cultivation area or natural vegetation. The second landscape unit, Figure 3 (b), was identified by a significant area of cultivation, and the central point where there were differences between the scale and the merger was to the urban area. This class is unique because of the different forms of the city and high radiance, dividing it into small segments making its analysis difficult. To gain better results the analysis of this class could be separated, however, when interpreted along with other classes will cause confusion, especially because of the image analysis Oriented Geographic Object.

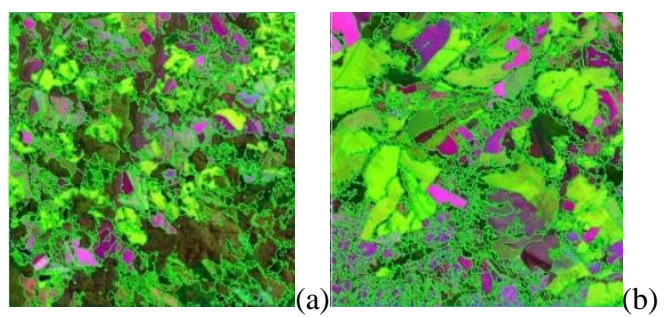

Figure 3 (a) and (b). Image ResourceSat Liss III Color combination representing the true-false color scale of 40 and melt 50 used in the first and second drive landscapes.

\subsection{Separate analysis of descriptors}

With the segmented image could identify differences in the face of the landscape, both in the areas of cultivation, vegetation, as in urban areas. Upon the rising of targeting vectors generated images with shape descriptors in a GIS program. This spatial distribution of shape descriptors allowed the analysis of each of the descriptors and the use and occupation of land in the two landscape units. Figure 5, the first landscape unit, exemplifies this fact, lighter shades and area value in smaller square meters but darker in large quantities and with greater numerical values. These areas are highlighted with arrows.

After these processes comparisons were made between the descriptors of shape attribute arising from AOO, seeking to identify characteristics that have a similar relationship to each other. We carried out the tests consistent in each of the descriptors. However, there were only identified six key combinations representing relations between its characteristics as follows: Area - long, medium circumference-factor, compactness-circumference, compactness form factor, length - convexity, solid - form factor.

\subsubsection{Area - Length}

Figures 4, 5, 6 and 7 show the fields of study, first and second units and the area and length of the descriptors. The analysis of these figures, identifies the brown shade areas are the larger regions comparing it with other areas. These fields range from $4565,06 \mathrm{~m}^{2}$ and $24591,07 \mathrm{~m}^{2}$ (Figure 4), to drive one and the values of $534,687.50 \mathrm{~m}^{2}$ and $2127187,50 \mathrm{~m}^{2}$ to 2 (Figure 6) with small regions shown by shades of yellow, ranging in the legend of $625,00 \mathrm{~m}^{2}$ and $136562,50 \mathrm{~m}^{2}$

In the case of length descriptors in Figures 5 and 7, the highest values are 2444.38 and $24591,07 \mathrm{~m}$ to the first unit in the north-south 
direction, following the Pitangui River. The second unit, 2906.48 to $14,624,64 \mathrm{~m}$ identifies places where there is the presence of large areas of cultivation or soil exposed back to agriculture. The southeast, of figure 7 , there is the urban area, where the regions are small and shown in the yellow color legend.

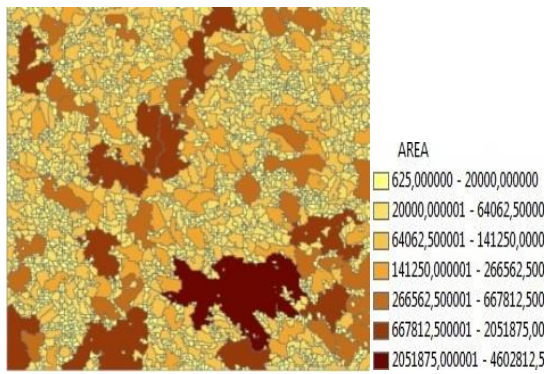

Figure 4 - Image descriptor area of the first unit landscape

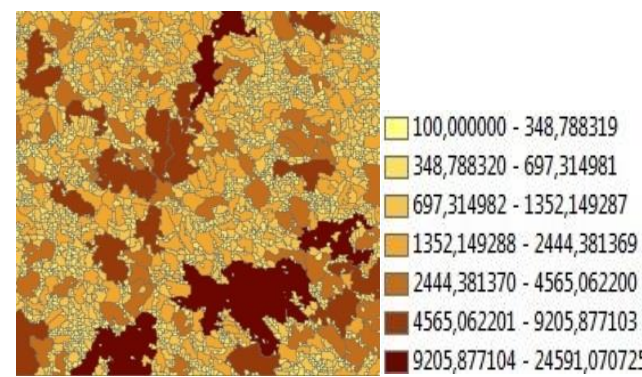

Figure 5 - Descriptor length of the first unit landscape

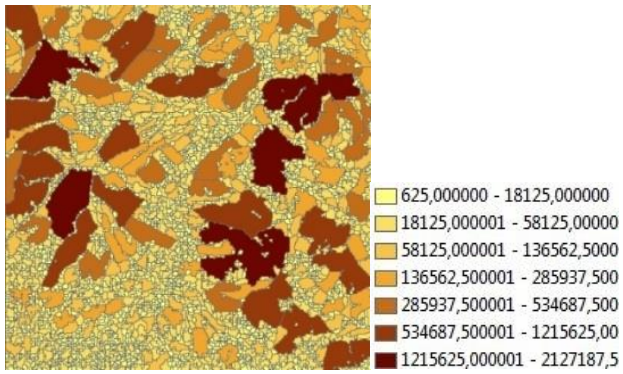

Figure 6 - Descriptor of the second image area landscape unit.

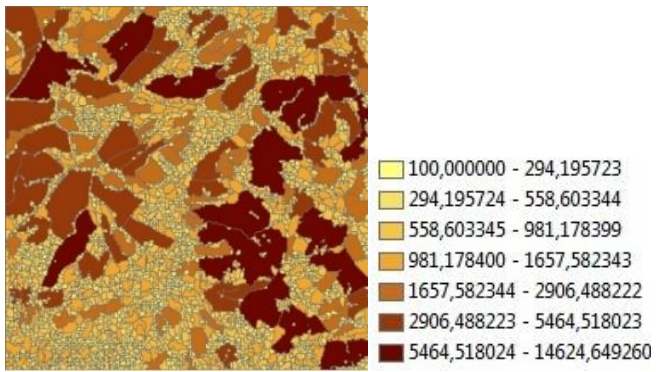

Figure 7. Descriptor of the second image area landscape unit

Figures 8, 9, 10 and 11 show the normalized images (area $\mathrm{x}$ length) and the respective scatter plots of the two landscape units. The Figure 8 and 9 refer to the unit 1 . In the Figure 9 graph, this information is apparent, since the points represented by the medium to dark brown tones are grouped together and grow in both of the $\mathrm{X}$ axis corresponding to the length descriptor. The shaft $\mathrm{Y}$-axis is corresponding to the descriptor area, having only a few points. If scattered in the picture, these correspond to the largest areas.

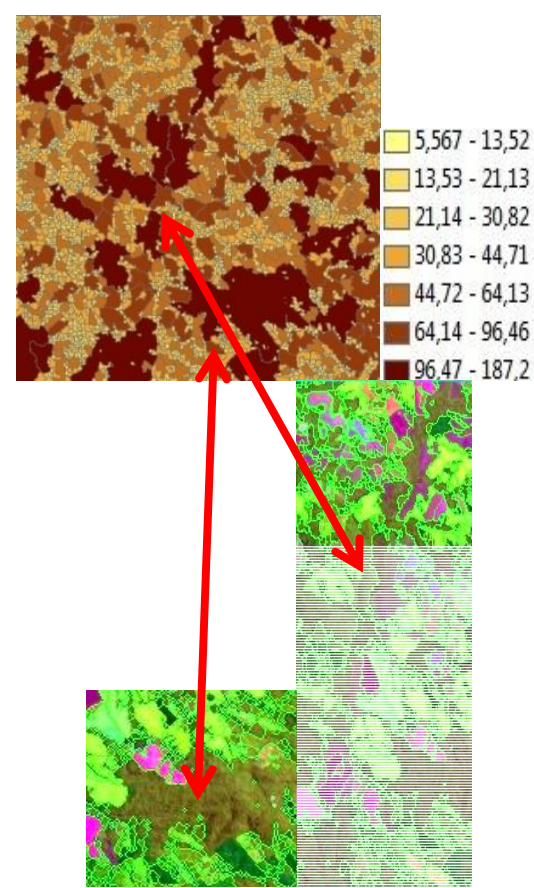

Figure 8 - Standard Image area and length, unit 1

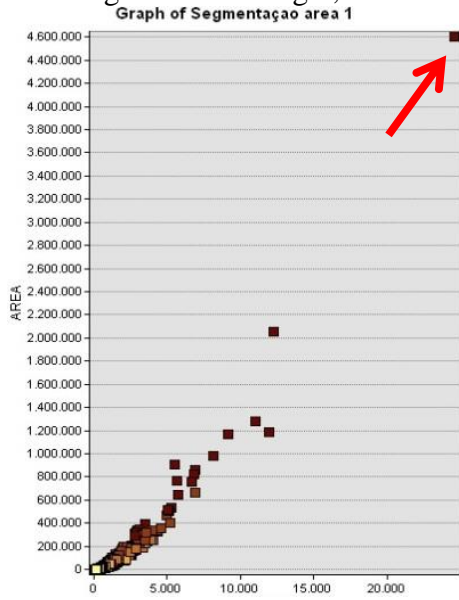

Figure 9 - Graph standard area and length, unit 1.

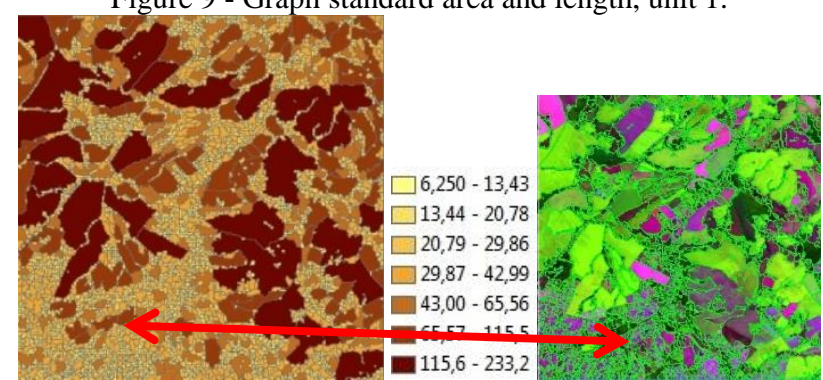

Figure 10 -Image normalized area and length, second landscape unit 


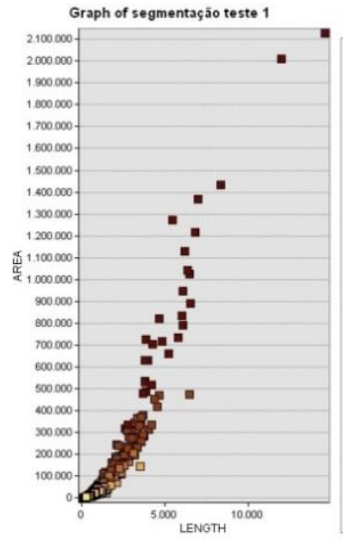

Figure 11 - Graphic standard area and length, the second landscape unit.

A peculiarity found in the first landscape unit, Figure 8 , is a green area (red arrow and inset) with high numerical value area and length area and has a high similarity between descriptors, highlighted in Figure 10 by the graph where the region appears isolated (red arrow).

In Figures 10 and 11 refer to the unit 2. In Figure 11 the normalized image area and length descriptors in brown shades areas are larger and extended. In Figure 11 these scatter graphs show the relation between these descriptors, the increase in size is dependent to the descriptors. The areas of lesser numerical value (red arrow) are in shades of yellow, indicating that they have no similarity in either area or length. Furthermore, they occupy less significant regions to the larger areas. The colored composition in detail also highlights these small areas concerning urban area (red arrow)

When comparing the two landscape units, the first to the second through the area and length of the descriptors, it is identified that the first landscape unit, exhibits small and long areas facing agriculture and vast and extended areas with the presence vegetation. In the second landscape unit, are identified large areas with significant lengths, focused on extensive farming, and small areas with small distances from targeting the urban area of the city of Ponta Grossa.

\subsubsection{Circumcised - Form Factor}

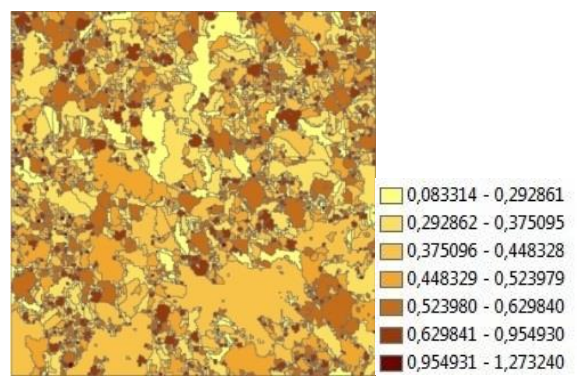

Figure 12. Picture referring to the descriptor circumcised, first landscape unit

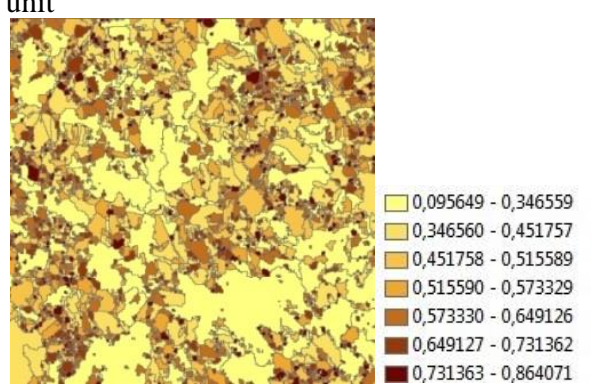

Figure 13 Picture is referring to the descriptor factor first landscape unit form.

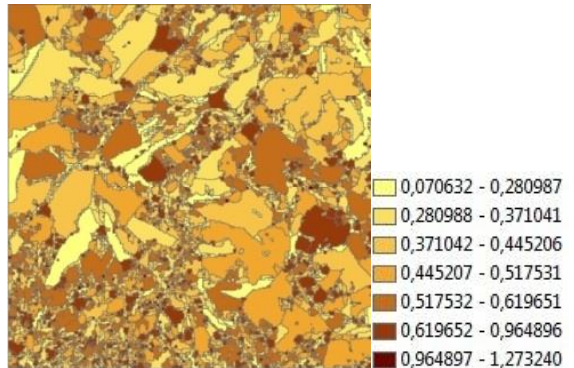

Figura14. Image related to the descriptor roundness second landscape unit.

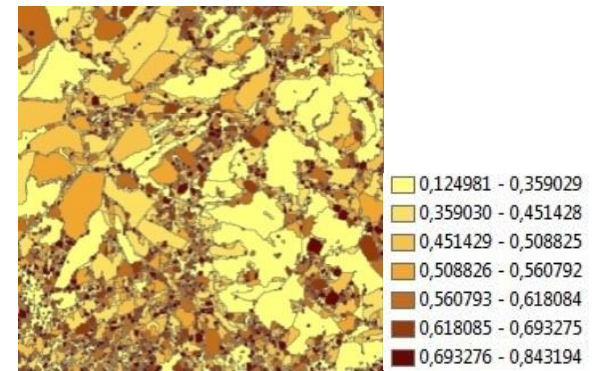

Figure 15. Picture referring to the descriptor formfactor second landscape unit.

Figures 12, 13, 14 and 15 are consistent first and second landscape units, referring to roundness and form factor descriptors. The normalized image roundness between descriptors and form factor are shown in Figures 16 and 18 and; comparative graphs between the two descriptors in Figures 17 and 19.

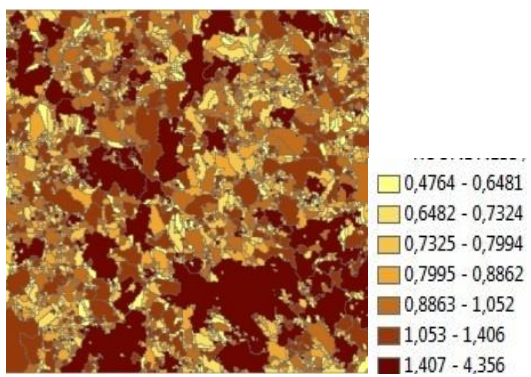

Figure 16. Normalized image among descriptors, unit one, descriptors roundness and formfactor.

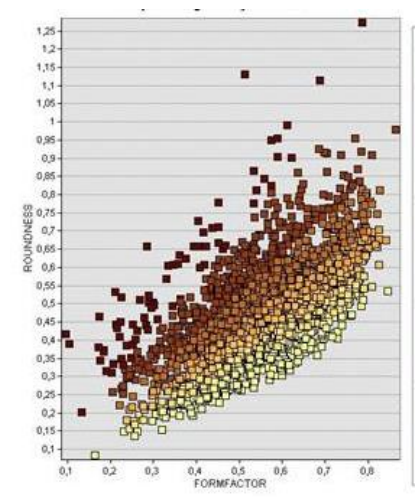

Figure 17 - graph of the normalized image among descriptors, unit one, descriptors Roundness and formfactor. 


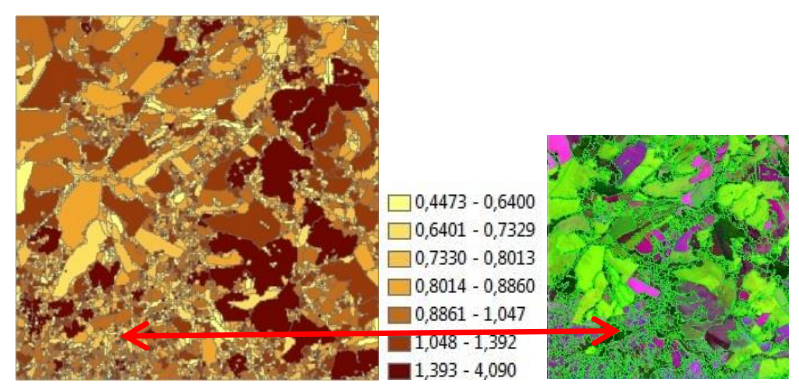

Figure 18. Normalized image among descriptors, unit 2 radius descriptors and form factor.

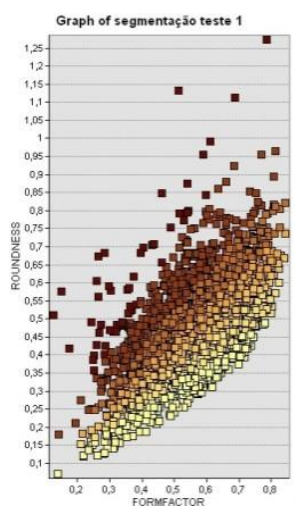

Figure 19. Graph of the normalized image among descriptors, second unit, roundness descriptors and form factor

Given that, the artificial images generated for each of the descriptors and the normalization of the same shows increasing values in the legend where the darkest color match the most significant areas in the descriptor and the lighter ones that have less significance. Notethat in radius image, Figure 12, the greatest radius values are few and scattered. These are highlighted by the color dark and medium brown, ranging in legend between the values of 0.629 and 1.273, as the segments with lower radius cover most of the landscape presenting itself in shades of yellow with values of 0.083314 and 0.375095 .

As for the form factor, figure 13 , it is identified that the greatest figures in shades of dark brown present their values between 0.649127 and 0.864071 and lighter shades of yellow varying between 0.095649 and $0,451,757$.

In figura 18 corresponding to normalized image, identifies that the largest areas present with greater value, highlighted by dark brown color because they are present in more significant amounts in the image.

In the graph, Figure 19, the linear growth between the two descriptors occurs mainly in the areas of smaller, presented in the medium brown color chart. Note the linear growth between the two descriptors as highlighted by the red line.

The large areas on the chart show up in yellow. It is evident that as the descriptor radius, the y- axis, increases the form descriptor factor. And therefore, the $\mathrm{x}$-axis also increases, but differently, causing disorder in and creating a parabola points on the graph

Another feature of these regions is that part of the transition areas between larger areas is that agricultural areas intercalate with areas of vegetation. Because of this, there are some objects identified in small spaces. By this analysis can be stated that in these areas are unclear segmentations caused by existing spectral confusion in the image, i.e., the significant amount of information in one pixel often caused by relief.

In mid-sized regions also the similarity between the descriptors can be identified, as demonstrated in the circled areas. Note that both the image related to radius as in the form factor, are areas similar in brown tones, and in the chart, this similarity is more evident when we note a linear growth between the comparison of these two descriptors, as indicated by the blue arrow.

Figure 14 relating to radius descriptor of the second landscape unit, small areas are identified and presented by dark brown tones, demonstrating that these had a greater scope with the descriptor ranging from 0.619652 and 1.273240 . There are areas where the radius descriptors were less comprehensive presented in yellow tones ranging from 0.070632 and 0.37041 .

Note that in this descriptor; significant regions were the small geared segments or urban area or areas of transition between the large crop fields.

Figure 15 regarding the form descriptor factor in the second landscape unit identified that just as in the radius descriptor the smaller areas were highlighted by shades of dark brown, ranging in legend between 0.618085 and 0.843194 . Larger areas were presented by the shades of yellow, ranging in legend between the values of 0.124981 and 0.451428 .

\subsubsection{Compactness - Form Factor}

Figures 21, 22, 23, and 24 show the first and second landscape unit. These images are descriptors reference to the compactness and form factor. Since the images $25,26,27$ and 28 represent respectively the normalized images and graphics relating to first and second landscape unit. There are 25 and 26 concerning the first landscape unit and 27 to 28 regarding the second landscape unit

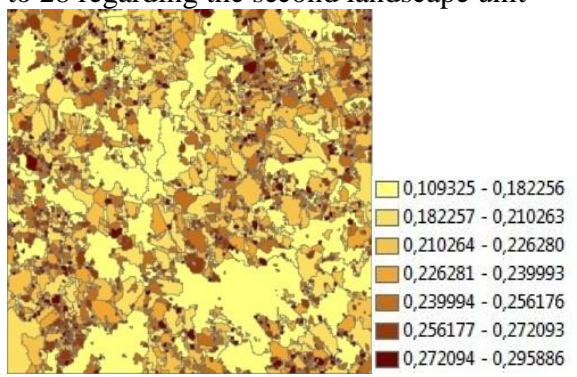

Figure 21. Concerning the first image landscape unit descriptor related to compactness.

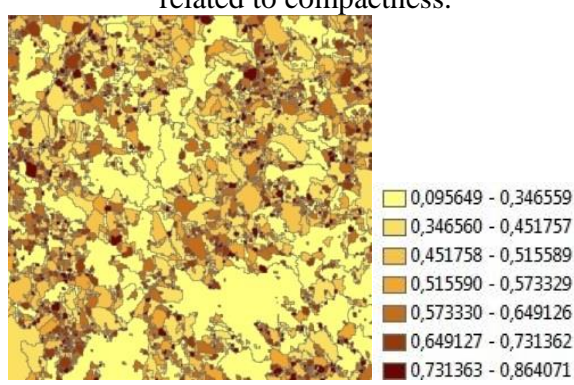

Figure 22. Picture regarding the first landscape unit referring to the descriptor form factor. 


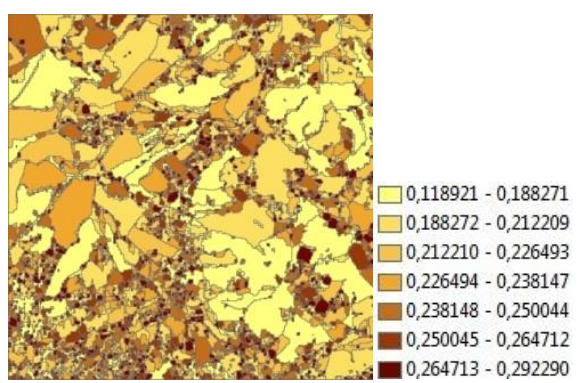

Figure 23. Concerning the second image landscape unit descriptor related to the compactness

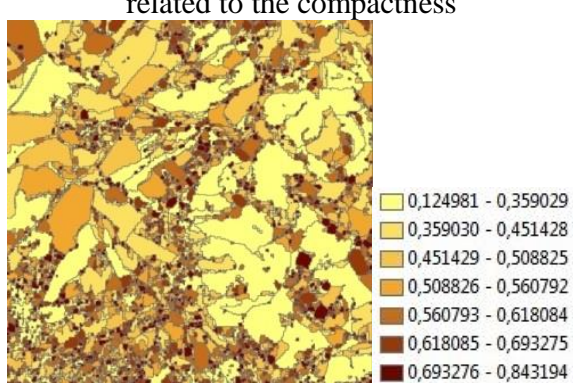

Figure 24. Picture referring the second landscape unit referring to the descriptor form factor.

As we have previously used the compactness and form factor descriptors have the smaller areas with medium brown tones to dark, as they present significant similarity with the descriptors. However in the first landscape unit identifies the presence of large segments of these that appear in shades of yellow, because they have no high compactness and forms defined as the smaller segments, which are presented in a circular fashion.

In the normalized image, Figure 25. It is observed that the smaller areas are shown in shades of yellow and larger areas are presented in dark brown tones. This is because of standardization, that is held direct division of the figures presented the descriptors legends, values referring to smaller areas, highlighted in artificial images from the descriptors by brown tones are now presented in shades of yellow, because the reason for the division is less than the ratio of the larger areas

In this graph in Figure 26, there is linear growth between the two descriptors. It rushes due to the similarity of form factor and compactness descriptors. Are noted by the graph linear growth occurs both in small areas highlighted by shades of dark brown, presented at the beginning of the graph between the values 0.1 and 0.2 for the $\mathrm{x}$-axis refers to the form descriptor factor and $0,11-.19$ descriptor for compactness prescribed on the $y$-axis. As for the areas which are in shades of yellow, they are $\mathrm{x}$-axis between 0,6 and 0,24 and the y-axis values between 0.8 and 0.29 .

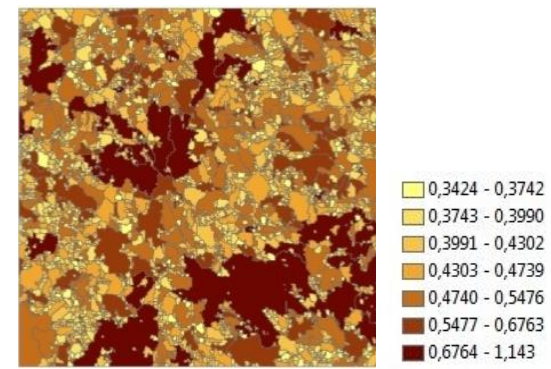

Figure 25. Normalized image regarding the first landscape unit regarding the descriptors compactness and formfactor.

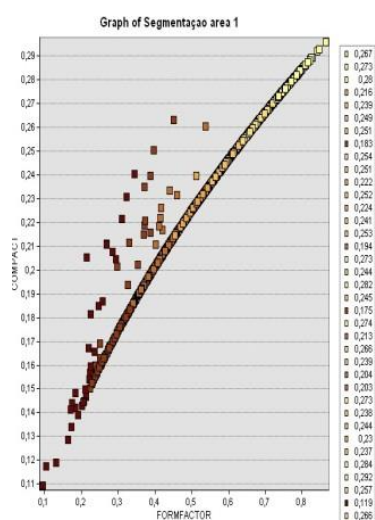

Figure 26. Comparative graph between compactness descriptors and form factor, regarding the first landscape unit.

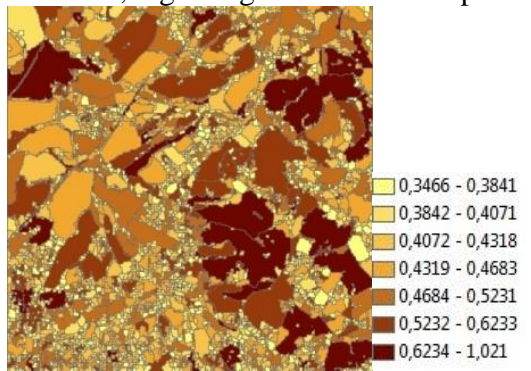

Figure 27. Normalized Image landscape on the second unit between the descriptors and compactness form factor.

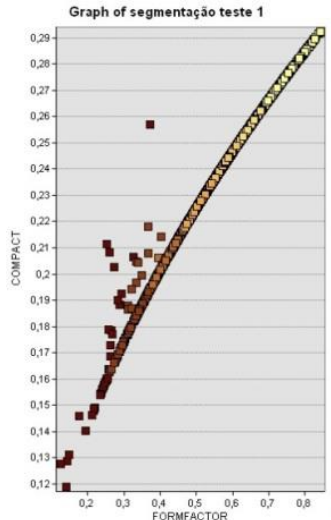

Figure 28.Comparative graph of compactness descriptors and form factor. Second landscape unit.

As previously used the descriptors of compactness and form factor presented in shades of dark brown small areas that have defined shapes and compactness in their spectral response.

It is noted in Figures 25 and 27 refer to normalized image descriptors. Vast areas presented in shades of dark brown to medium brown are because to the fact these are shown to be the defining descriptor form of the image.

In Figures 26 and 28, about the graphics between compactness descriptors and form factor. We noted that shears are directly proportional increasing between the descriptors, i.e., the extension in which the areas became more compact performing as defined.

Note that in the graph based on the $\mathrm{X}$ axis values between 0.0 and 0.2 and $y$-axis values between 0.12 and 0.2 , are the areas in the image for each descriptor are shown in shades of dark brown.

The areas which are in shades of yellow, which refer to larger areas are $\mathrm{x}$-axis values between 0.6 and 0.8 and the $\mathrm{Y}$ axis values between 0 and 0.2429 . 
While comparing these descriptors, it is noted that in both the first area of study and in the second area of study, the descriptors compactness and form factor, are presented in shades of brown. Smaller areas make references to regions where there are little areas of cultivation and vegetation. The case of the first landscape unit, and the second landscape unit such small areas are mainly reference the urban area of Ponta Grossa and the small areas between the vast areas intended for agribusiness.

\section{Conclusion}

The Object-Oriented Analysis made possible to subdivide the landscape units into segments discriminating vectors for each segment database. This study enabled better analysis of the regions and landscape morphology, considering that the descriptors were used in fashion attribute.

When comparing all descriptors, the conclusion is that sound descriptors, form factor, compactness and radius descriptor primarily emphasize small areas because they have rounded shapes. As the descriptors area, length and curvature, these are suitable for large stress regions.

It was identified in the first landscape unit, north-west of Alagados Dam, the largest portions of land are from dense and sparse vegetation considering that these are distributed mainly along the river course, where regions susceptible to flooding. These areas were highlighted particularly by the descriptors area, convexity and length.

In this same landscape unit still identified by the factor, descriptors way radius descriptor, robustness and compactness, smaller areas related to small properties linked to family farming, and even areas with a large amount of spectral confusion regarding the transition areas agriculture regions with regions of vegetation in its two stages of development.

In the second landscape unit used in the present study, located westcentral Alagados Dam, the descriptors area, length and convexity, it was of paramount importance for the identification of large tracts of land geared toward agribusiness.

It is identified through this study that beyond the areas facing agriculture, and urban area of Ponta Grossa there is significant sound descriptors, compactness, radius and form factor. It highlights small areas due to a large amount of information from the reflectance of the urban area. It was subdivided into small areas as the spectral response of object.

Other important points in this landscape unit were to exchange between regions aimed at agriculture, which mainly identifies the presence of exposed soil and vegetation in the process of AOO, subdivided these areas in small arrays, also due to spectral confusion.

Consequently, the landscape morphology in both areas of study proposals is summarized as:

The first area, located north-west of Alagados Dam, is identified mainly by medium regions to mostly small farms focused on family farming and larger areas dominated by vegetation.

The second area, located west central Alagados Dam, identifies large areas related to extensive agriculture, intended mainly for the cultivation of soybeans and wheat, also determines the presence of small amounts of vegetation, which are derived from Riparian forests and small rivers that cross this region, and exchange between the properties.

\section{Referencias}

